\title{
Ultrasound-guided percutaneous injection of triamcinolone acetonide for treating capsular contracture in patients with augmented and reconstructed breast
}

\author{
Luca Maria Sconfienza • Carmelina Murolo • Simone Callegari • Massimo Calabrese • \\ Edoardo Savarino • Pierluigi Santi • Francesco Sardanelli
}

Received: 28 May 2010/Revised: 29 June 2010/Accepted: 25 July 2010/Published online: 9 August 2010

(C) European Society of Radiology 2010

\begin{abstract}
Objectives To evaluate ultrasound (US)-guided treatment of capsular contracture (CC) in patients with reconstructed/ augmented breast.

Methods Twenty-five patients with grade IV CC were treated with peri-implant US-guided injection of triamcinolone acetonide. Before/after treatment, maximum capsular thickness (MCT) was measured by ultrasound and pain assessed with visual analogue score (pain-VAS). Patients with pain relief at 1 month were considered early responders (ERs). Another injection was performed in patients without pain relief at 1 month (late responders, LRs).
\end{abstract}

L. M. Sconfienza

Facoltà di Medicina e Chirurgia,

Scuola di Specializzazione in Radiodiagnostica,

Università degli Studi di Genova,

Viale Benedetto XV 6,

16132 Genova, Italy

\section{Murolo}

Struttura Semplice di Radiologia Interventistica,

Struttura Complessa di Diagnostica per Immagini,

IST Istituto Nazionale per la Ricerca sul Cancro,

Largo Rosanna Benzi 10,

16132 Genova, Italy

\section{S. Callegari $\cdot$ P. Santi}

Dipartimento di Scienze Chirurgiche e Diagnostiche Integrate, Università degli Studi di Genova,

Struttura Complessa di Chirurgia Plastica e Ricostruttiva,

IST Istituto Nazionale per la Ricerca sul Cancro,

Largo Rosanna Benzi 10,

16132 Genova, Italy
Results One patient (treated with chemo-radiotherapy) experienced severe pain and local reaction after the second injection, requiring surgery. Twenty-four patients had baseline MCT of $1.8 \pm 0.3 \mathrm{~mm}$ and pain-VAS of $4.9 \pm 0.5$, the baseline MCT of 19 ERs $(1.7 \pm 0.2 \mathrm{~mm})$ being significantly lower than that of 5 LRs $(2.1 \pm 0.2 \mathrm{~mm})(p=0.030)$. ERs had significantly reduced MCT and pain-VAS at one $(1.1 \pm 0.3 \mathrm{~mm} ; 1.5 \pm 0.5)$ and 6 months $(1.1 \pm 0.2 \mathrm{~mm} ; 0.9 \pm 0.7$, respectively) $(p<$ $0.001)$. At 1 month, LRs had a significantly reduced MCT $(1.6 \pm 0.1 \mathrm{~mm}, p=0.042)$ but non-significantly changed painVAS (4.7 \pm 0.2$) ; 5$ months later, MCT reached $1.0 \pm 0.1 \mathrm{~mm}$, pain-VAS reached $0.8 \pm 0.5(p<0.044)$. Significant correla-

\footnotetext{
M. Calabrese

Struttura Complessa di Diagnostica Senologica,

IST Istituto Nazionale per la Ricerca sul Cancro,

Largo Rosanna Benzi 10,

16132 Genova, Italy

E. Savarino

Dipartimento di Medicina Interna, Università degli Studi di Genova,

Viale Benedetto XV 6,

16132 Genova, Italy

F. Sardanelli

Dipartimento di Scienze Medico-Chirurgiche,

Università degli Studi di Milano, Unità di Radiologia,

IRCCS Policlinico San Donato,

Via Morandi 30,

20097 San Donato Milanese, Milano, Italy

L. M. Sconfienza ( $₫)$

Unità di Radiologia, IRCCS Policlinico San Donato,

Via Morandi 30,

20097 San Donato Milanese, Milano, Italy

e-mail: io@lucasconfienza.it
} 
tion between the relative variation of MCT and pain-VAS (1 month/baseline) was found.

Conclusions US-guided injection of triamcinolone acetonide is effective in treating grade IV CC.

Keywords Ultrasound guidance - Capsular contracture · Breast . Triamcinolone acetonide

\section{Introduction}

Capsule formation around a breast implant is the effect of a low-grade chronic inflammatory reaction to the foreign body, resulting in a layer of fibrous dense connective tissue [1]. Normally not thicker than $1 \mathrm{~mm}$ [2], the capsule is a part of the normal healing process and may help in keeping the implant in situ $[3,4]$.

Capsular contracture (CC) is the most frequent complication after breast augmentation or reconstruction with an implant [5]. The incidence during the first year after surgery ranges from $2 \%$ to $30 \%$ [6]. CC is mostly unilateral and seems to be influenced by several factors, such as patient age, type of implant (shape, surface and material), surgical technique, and occurrence of postoperative complications [5]. In addition, the presence of a bacterial microfilm on the surface of the implant seems to be a favouring factor [7]. In 1978, Baker [8] described four clinical stages of CC. Stage I represents a normal breast. Stage II represents a breast that is a little firmer than it should be but still looks normal. Stage III represents a very firm and aesthetically abnormal breast. Finally, stage IV represents an aesthetically abnormal and painful breast.

In the past, breast implants had a smooth surface. More recently, new implants with textured surface were introduced in clinical practice, but their role in reducing $\mathrm{CC}$ remains controversial [5]. In addition, women who underwent radiation therapy have a higher incidence of $\mathrm{CC}$ $[9,10]$.

Over the past few decades, several therapies for CC have been proposed. In patients with mild symptoms, the disease can be managed conservatively with massages or tight immobilisation [4]. An alternative therapeutic approach is to perform an open [11] or closed capsulotomy or a capsule excision $[12,13]$. Recent papers described CC improvement after oral administration of leukotriene receptor antagonists [6]. Finally, steroid injection has been demonstrated to be effective for the treatment of this condition [14-16]. The injection technique proposed by Caffee [15] consists of a three-step procedure that implies the use of a needle, a catheter, and a custom-fabricated electrosurgery probe without any image guidance.

The purpose of our study was to describe an ultrasound (US)-guided technique to inject steroid between the capsule and the implant as well as to evaluate the efficacy of this procedure in patients with $\mathrm{CC}$.

\section{Methods and materials}

Study population

This study had local Ethics Committee approval and all patients provided informed consent. The current report concerns the 6-month outcome of 25 consecutive patients with reconstructed or augmented breast affected by Baker grade IV CC [8], who were treated with a local US-guided injection of steroid at our Institution from March 2003 to December 2006, and the presence of a stage IV CC, according to Baker [8].

Patients with reconstruction were implanted monolaterally with anatomical or round-shaped textured implants or breast expanders (Mentor Corp., Santa Barbara, CA, USA or Inamed Corp., Santa Barbara, CA, USA). Patients with augmentation were implanted bilaterally with anatomical textured implants (Mentor Corp., Santa Barbara, CA, USA or Inamed Corp., Santa Barbara, CA, USA).

We treated one breast in each patient. Demographics and population features are shown in Table 1.

The visual analogue score (pain-VAS) was used for pain assessment [17]. It consisted of a $10-\mathrm{cm}$ graduated scale from 0 (no pain) to 10 (unbearable pain): the patient marks the point she feels to correspond to her pain [17].

\section{Pre-treatment US evaluation}

Before treatment, we performed a US examination to assess the capsular thickness, the implant and the pouch of the affected breast (Fig. 1). Maximum thickness of the capsule and its location was recorded.

US-guided procedure

The study was conducted with the following US equipment: a 5000 HDI (ATL Electronics, Koninklijke Philips Electronics, Eindhoven, the Netherlands) provided with a high-resolution broadband linear array transducer (5-12 MHz). US-guided procedures were performed by one radiologist with 25 years' experience in breast and interventional US (C.M.). A second operator was necessary to inject the drug during the procedure. To avoid any movement during the procedure and to prevent the consequence of possible vagal reactions, the patients lay in a supine position.

The probe was cleaned with a non-alcoholic solution of didecyldimethylammonium chloride $0.45 \%$ for at least $2 \mathrm{~min}$ to ensure complete sterilisation. The skin was cleaned with a solution of iodopovidone $10 \%$ (or 


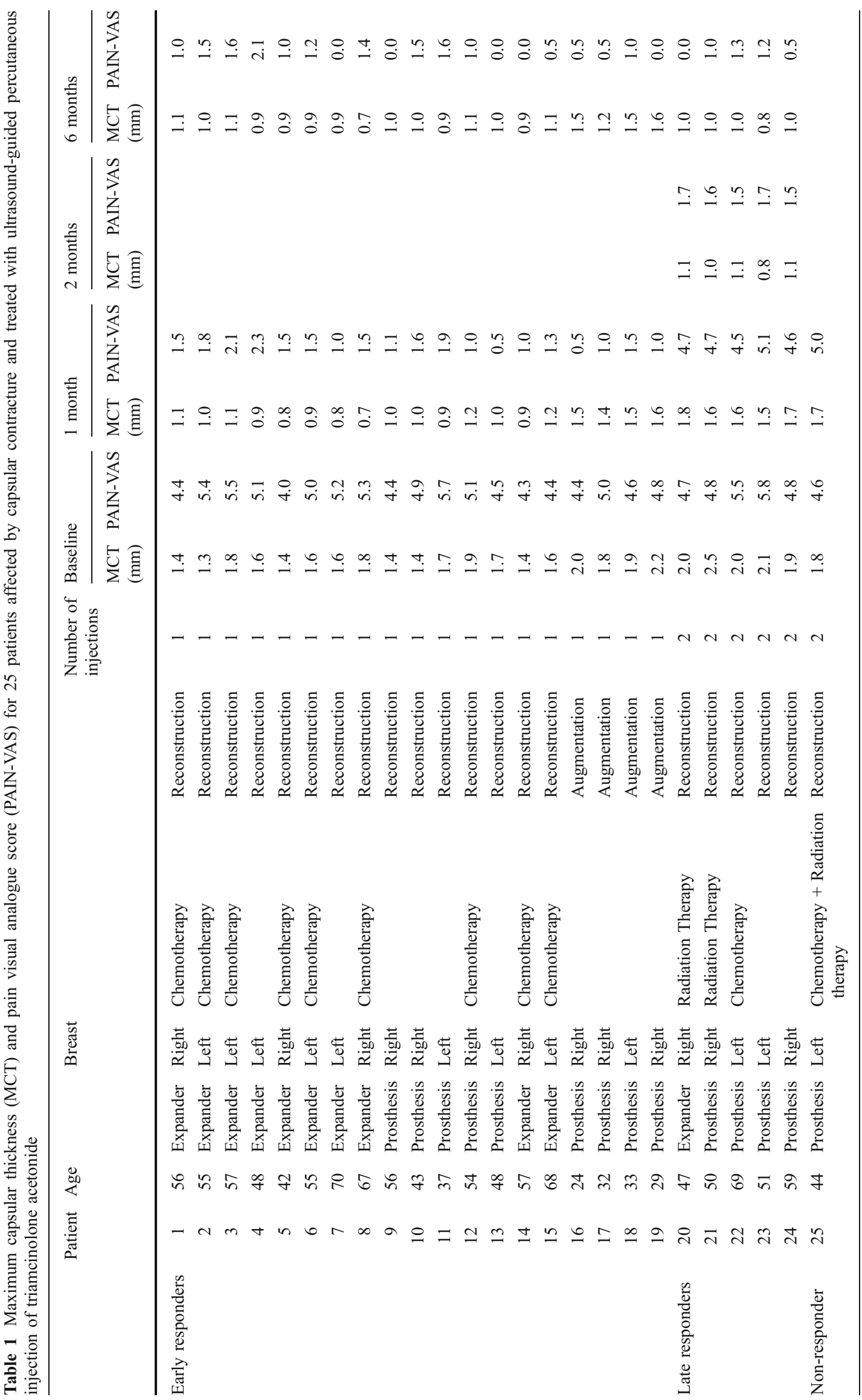




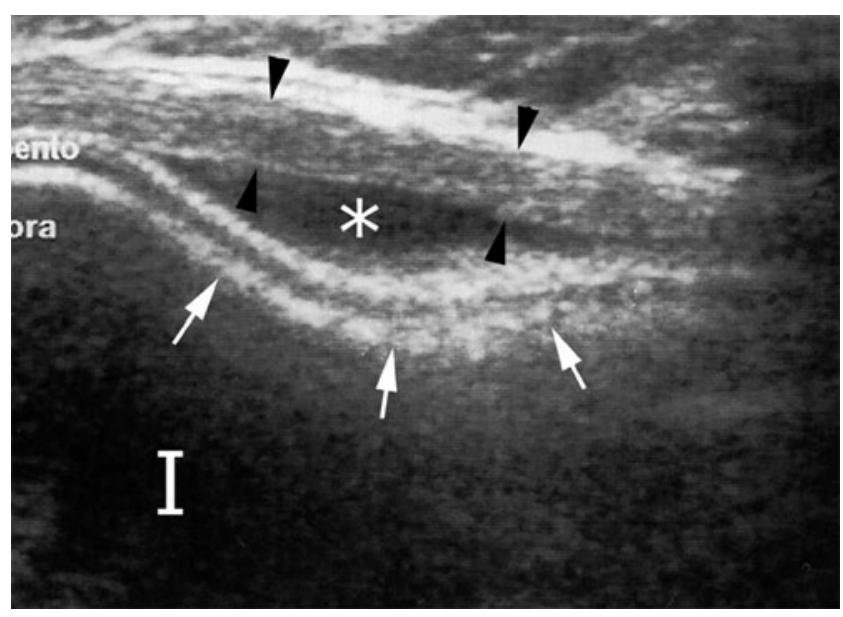

Fig. 1 A 43-year-old woman with capsular contracture (black arrowheads) in her reconstructed and implanted right breast. A small fluid collection in an implant folding is clearly visible (asterisk). White arrows $=$ double-layer implant surface; $\mathrm{I}=$ anechoic echotexture of the implant

benzalkonium chloride $0.25 \%$ when allergy to iodine was reported by the patient) and then draped with sterile tissues. With ultrasound, the operator localised a small folding of the implant in order to have a safe site to introduce the needle. Sterile lubricant contact gel between the probe and the skin was used (Glissen ${ }^{\circledR} 12.5 \mathrm{~g}$, Pharm. Fabrik Montavit Ges.m.b.H., Absam, Germany). In 3 out of 6 patients with a breast expander, this device was slightly emptied to make more space for the injection. US guidance was used to introduce a 22-gauge needle between the contracted capsule and the implant. The needle was introduced coaxially to the US probe with an inclination of $45^{\circ}$ (Figs. 2 and 3a) and attention was paid to the tip location in the periprosthetic space, being careful

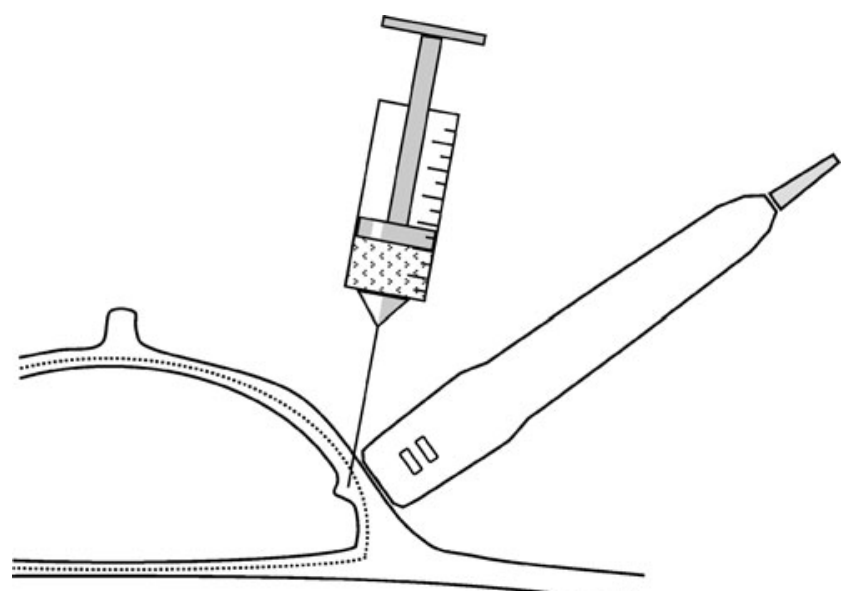

Fig. 2 Schematic drawing of the procedure. The needle tip is inserted into a small folding of the implant under ultrasound guidance. Dotted line $=$ contracted capsule
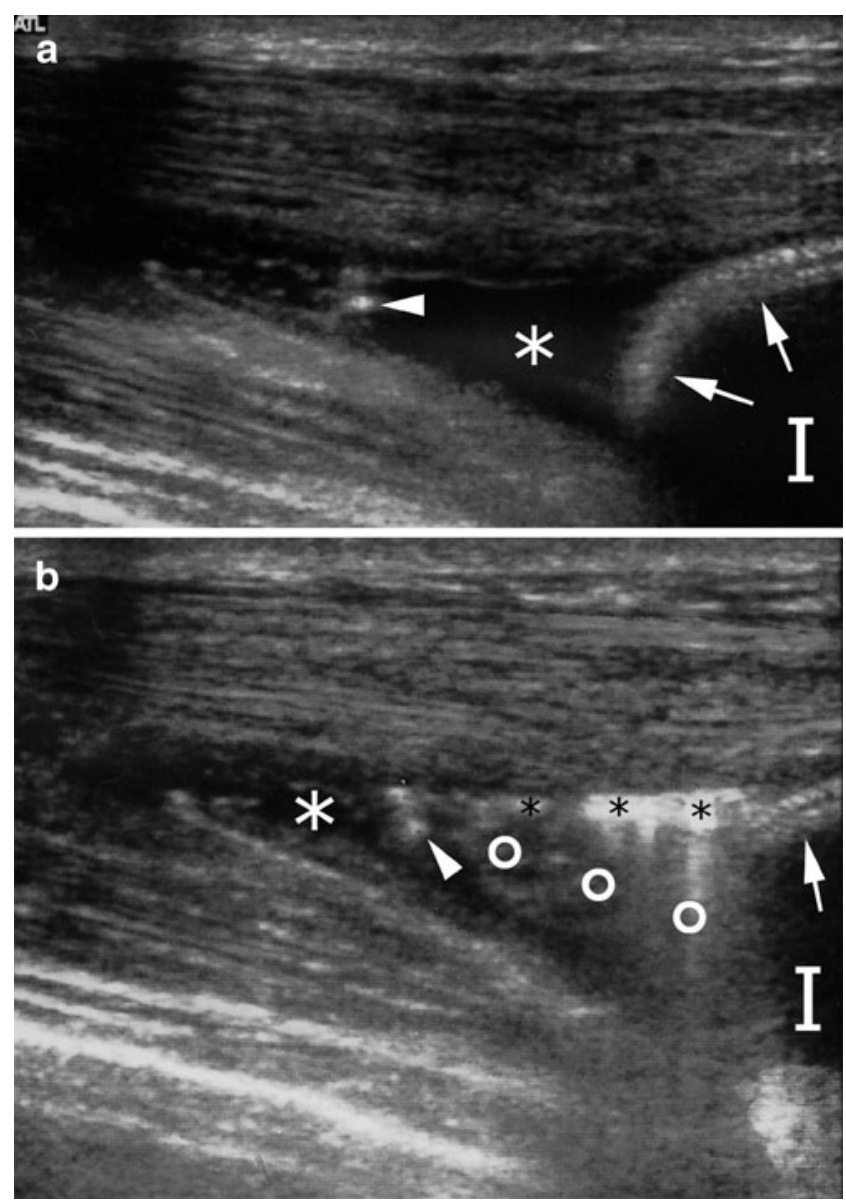

Fig. 3 A 24-year old woman with capsular contracture in her augmented right breast. (a) Needle tip (arrowhead) positioning in a small folding (asterisk) of the implanted breast (I). White arrows $=$ double-layer implant surface. (b) Steroid is injected (circles) into a small folding (asterisk) of the implanted breast (I). Needle tip (arrowhead) is visible and correctly positioned. Note the small amount of air (small black asterisks) injected together with the drug. White arrow $=$ double-layer implant surface

to avoid any contact between the needle tip and the implant surface. Thereafter, $1 \mathrm{~mL}$ of triamcinolone acetonide (Kenacort $40 \mathrm{mg} / \mathrm{mL}$, Bristol-Myers Squibb S.p.A., Italy) diluted in $10 \mathrm{~mL}$ of saline solution was injected (Fig. 3b). Finally, the needle was extracted, the skin cleaned and a slightly compressive medication was applied. Patients were kept in observation for about $30 \mathrm{~min}$ and then discharged from the Department. Treatment protocol did not include any scheduled drug administration after patients' discharge. It was suggested that they apply some ice to the treated breast for $4 \mathrm{~h}$ to $6 \mathrm{~h}$ after the procedure. Each patient was instructed to massage the treated breast hourly during the first day and six times per day for 15 days to favour an homogeneous distribution of the drug around the implant.

At the 1 month time-point, patients who did not obtain a satisfactory reduction of symptoms were treated again with 
a second injection, with the same technique already described.

Follow-up

Ultrasound evaluation of capsular thickness as well as clinical evaluation with pain-VAS score was performed 1 and 6 months after the first treatment. Patients who received a second injection were also re-evaluated 1 month after the second treatment.

\section{Statistical analysis}

Patients who received one injection were considered early responders (ER), whereas those who received two injections were considered late responders (LR).

For both ER and LR, values of capsular thickness or painVAS score at baseline were compared with those obtained at 1 month or at 6 months using Wilcoxon's test. The correlation between capsular thickness and pain-VAS score at baseline, at 1 month and at 6 months was calculated for ER, LR and overall. Similarly, the correlation between the absolute and relative variation of both capsular thickness and pain-VAS score between baseline and 1 month was calculated.

Values of capsular thickness at baseline in ER were compared with those of LR using the Mann-Whitney $U$ test.
For statistical analyses, software SPSS version 17 (SPSS, Chicago, IL, USA) was used. A $p$ value equal to or less than 0.05 was considered significant.

\section{Results}

All patients had a submuscular breast implant and were operated within 12 months from the occurrence of CC.

Steroid injection between capsule and implant was performed in all patients. No implant perforation occurred. In one patient with breast reconstruction, who had just completed a cycle of chemotherapy and radiation therapy, a severe pain and local reaction occurred after the second injection. She then underwent an urgent operation to surgically remove the implant.

Out of the remaining 24 patients, 19 were ER and 5 LR. Results regarding the treated breasts are reported in Tables 1 and 2 and graphically represented in Fig. $4 \mathrm{a}$ and $\mathrm{b}$.

At baseline, maximum capsular thickness of 19 ER $(1.7 \pm 0.2 \mathrm{~mm})$ was significantly lower than that of five LR $(2.1 \pm 0.2 \mathrm{~mm})(p=0.030)$. Compared to baseline, ER had significantly reduced maximum capsular thickness and painVAS at 1 and 6 months. Compared to baseline, at 1 month, LR had a significantly reduced maximum capsular thickness but a non significantly changed pain-VAS. At 6 months (5 months after the second injection), maximum capsular thickness and

Table 2 Results of ultrasound-guided treatment of capsular contracture in 25 patients

\begin{tabular}{|c|c|c|c|c|c|c|c|}
\hline & & \multicolumn{2}{|c|}{ Early responders } & \multicolumn{2}{|c|}{ Late responders } & \multicolumn{2}{|l|}{ Overall $^{\mathrm{a}}$} \\
\hline & & Values & $\begin{array}{l}p \text { compared } \\
\text { with baseline }\end{array}$ & Values & $\begin{array}{l}p \text { compared } \\
\text { with baseline }\end{array}$ & Values & $\begin{array}{l}p \text { compared } \\
\text { with baseline }\end{array}$ \\
\hline \multicolumn{2}{|l|}{ Number of patients } & \multicolumn{2}{|l|}{19} & \multicolumn{2}{|l|}{5} & \multicolumn{2}{|l|}{25} \\
\hline \multirow[t]{2}{*}{ Age } & Mean \pm standard deviation & \multicolumn{2}{|l|}{$49 \pm 13.4$} & \multicolumn{2}{|l|}{$55 \pm 8.9$} & \multicolumn{2}{|l|}{$50 \pm 12.5$} \\
\hline & Median & \multicolumn{2}{|l|}{54} & \multicolumn{2}{|l|}{51} & \multicolumn{2}{|l|}{51} \\
\hline Breast & Right/left & \multicolumn{2}{|l|}{$10 / 9$} & \multicolumn{2}{|l|}{$3 / 2$} & \multicolumn{2}{|l|}{$13 / 12$} \\
\hline \multicolumn{2}{|c|}{ Chemotherapy/radiation therapy } & \multicolumn{2}{|l|}{$9 / 0$} & \multicolumn{2}{|l|}{$1 / 2$} & \multicolumn{2}{|l|}{$11 / 3$} \\
\hline \multicolumn{2}{|l|}{ Reconstruction/augmentation } & \multicolumn{2}{|l|}{$15 / 4$} & \multicolumn{2}{|l|}{$5 / 0$} & \multicolumn{2}{|l|}{$21 / 4$} \\
\hline Implant & Prosthesis/expander & \multicolumn{2}{|l|}{$9 / 10$} & \multicolumn{2}{|l|}{$4 / 1$} & \multicolumn{2}{|l|}{$14 / 11$} \\
\hline \multirow{4}{*}{$\begin{array}{l}\text { Maximum capsular } \\
\text { thickness }(\mathrm{mm})\end{array}$} & Baseline & $1.7 \pm 0.2$ & - & $2.1 \pm 0.2$ & - & $1.8 \pm 0.3$ & - \\
\hline & 1 month & $1.1 \pm 0.3$ & $<0.001$ & $1.6 \pm 0.1$ & 0.042 & $1.2 \pm 0.3$ & $<0.001$ \\
\hline & 2 months & - & - & $1.0 \pm 0.1$ & 0.042 & - & - \\
\hline & 6 months & $1.1 \pm 0.2$ & $<0.001$ & $1.0 \pm 0.1$ & 0.042 & $1.0 \pm 0.2$ & $<0.001$ \\
\hline \multirow[t]{4}{*}{ Pain visual analogue score } & Baseline & $4.8 \pm 0.5$ & - & $5.1 \pm 0.5$ & - & $4.9 \pm 0.5$ & - \\
\hline & 1 month & $1.5 \pm 0.5$ & $<0.001$ & $4.7 \pm 0.2$ & 0.068 & $2.2 \pm 1.6$ & $<0.001$ \\
\hline & 2 months & - & - & $1.6 \pm 0.1$ & 0.043 & - & - \\
\hline & 6 months & $0.9 \pm 0.7$ & $<0.001$ & $0.8 \pm 0.5$ & 0.043 & $0.9 \pm 0.6$ & $<0.001$ \\
\hline
\end{tabular}

All $p$ values were calculated using Wilcoxon's test

a Data reported in the "Overall" column include also the non-responder patient 


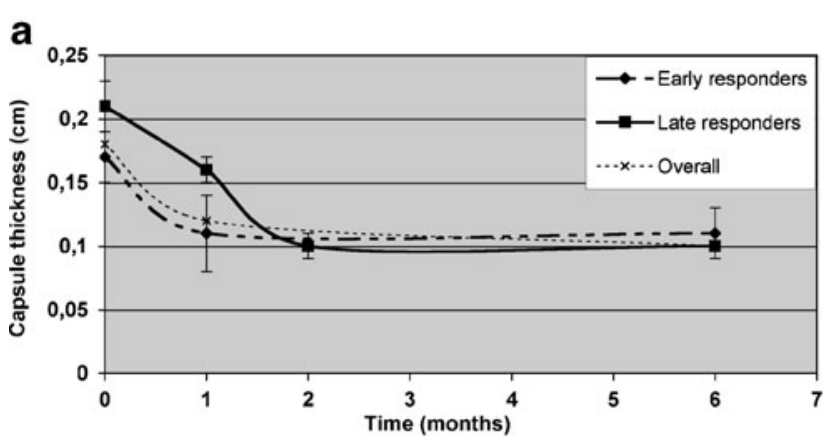

Fig. 4 (a) Evolution of maximum capsular thickness in 25 patients affected by capsular contracture and treated with ultrasound-guided percutaneous injection of triamcinolone acetonide. (b) Evolution of

pain-VAS of LR were significantly reduced in comparison with baseline values.

No significant correlation was found for ER, LR and overall $(p>0.153)$ between capsular thickness and painVAS score at baseline, at 1 month and at 6 months. Significant correlation between relative variation of maximum capsular thickness and pain-VAS (1 month versus baseline) was found $\left(R^{2}=0.178, p=0.040\right)$ (Fig. 5).

\section{Discussion}

Our experience demonstrated that US-guided injection of $40 \mathrm{mg}$ of triamcinolone acetonide in the peri-implant pouch of women with augmented or reconstructed breast affected by Baker grade IV CC is effective in reducing capsular thickness and patients' discomfort, $80 \%$ of patients being ER.

Capsular contracture is the most common complication in patients with breast implants. Over the years, its incidence has decreased. This reduction has been attributed to the use of new saline-filled implants and to the submuscular placement of the implants, while the role of textured implants is debated [5].

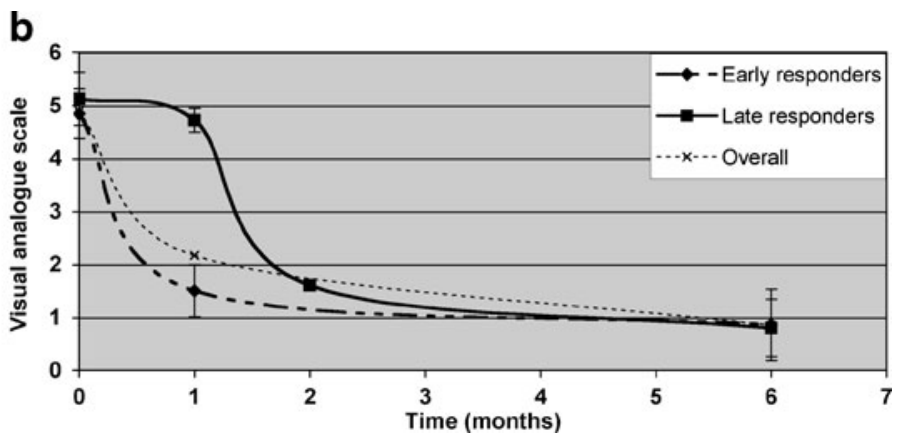

pain measured with visual analogue score in 25 patients affected by capsular contracture and treated with ultrasound-guided percutaneous injection of triamcinolone acetonide

Treatment of CC has been extensively discussed, but there is still no agreement on a generally accepted therapeutic pathway. Open and closed capsulotomy have been described as effective, but they represent a traumatic approach and results reported in the literature are discordant [4]. The anecdotal use of oral leukotriene receptor antagonists for treating $\mathrm{CC}$ has also been described [6]. These drugs seem to be quite effective. However, this still represents an off-label practice and further studies are required to validate the efficacy of this approach.

In our study, we included only grade IV CC because this stage is painful and makes pain treatment necessary.

The significantly lower capsular thickness at baseline between ER $(1.7 \pm 0.2 \mathrm{~mm})$ and LR $(2.1 \pm 0.2 \mathrm{~mm})$ seems to be an important point to focus on. These data may imply that a capsular thickness of about $2 \mathrm{~mm}$ is a threshold to determine whether a woman will need one or two injections. However, although the difference is statistically significant, this result should be tempered by the small number of patients included in the LR group.

The absence of correlation between capsular thickness and pain-VAS values as well as the almost-borderline correlation between the reduction in their relative values
Fig. 5 Correlation between the relative variation in the visual analogue score and the relative variation of maximum capsular thickness between baseline and 1 month in 25 patients affected by capsular contracture and treated with ultrasound-guided percutaneous injection of triamcinolone acetonide

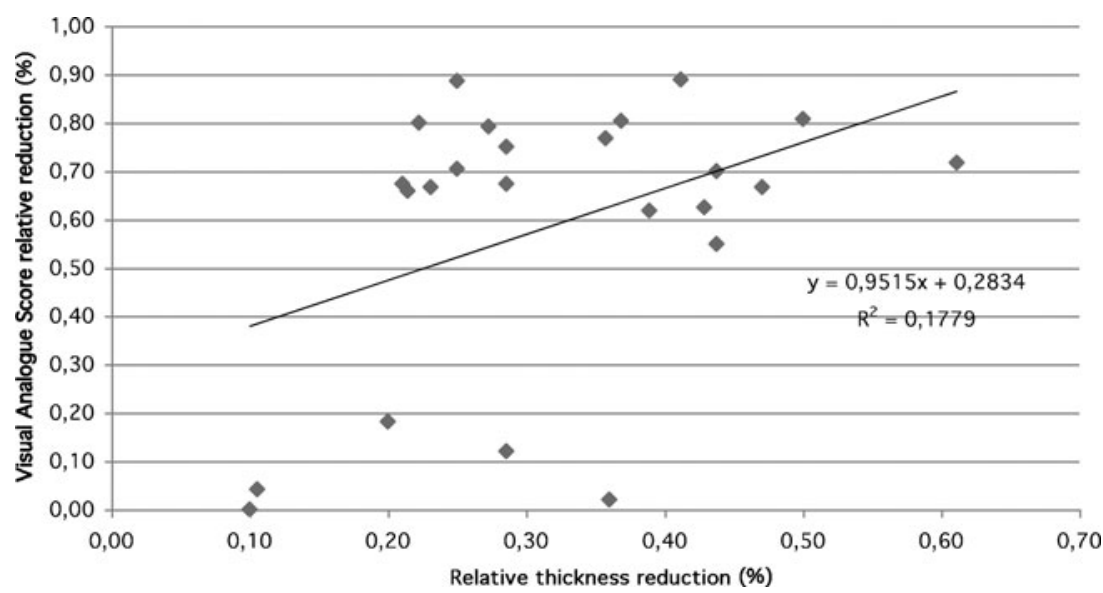


indicates that those parameters do not vary together. We could speculate that steroid has a faster and more effective action on pain rather than on capsular thickness. Only a longer follow-up may demonstrate a further reduction in this latter parameter. However, no data are available to support such a hypothesis.

We experienced one severe complication. This occurred after the second injection in a patient who had undergone combined chemotherapy and radiation therapy, and was severely debilitated. We decided to treat this patient because of an extremely painful CC. We can speculate that her general condition favoured the occurrence of a local reaction with severe pain, requiring removal of the whole implant. Notably, none of the patients who had undergone chemotherapy alone $(n=10)$ or radiation therapy alone $(n=2)$ experienced any adverse reaction.

We did not experience any complications related to the injection technique itself. However, the operator who performed the treatment had about 25 years' experience in breast and interventional US. Operators inexperienced in US-guided procedures could produce dissimilar results.

Compared with the injection technique described by Caffee and Rotatori [14], our US-guided treatment seems to have several advantages. First, the use of a simple 22-gauge needle seems to be less complicated than the combined used of a needle-catheter and a custom-fabricated electrosurgical probe. In addition, thin access produced by a fine needle is less prone to infections, compared with catheter access. Second, costs are another important factor to consider. Excluding money due for operators, drugs and room occupancy (that can be assumed comparable), the cost of a simple syringe and a 22-gauge needle are far lower than the cost of a needle-catheter and a custom-fabricated electro-surgical probe together with power generator. Third, the US guidance allows for real-time monitoring of the course of the needle through the skin and the capsule, enabling the operator to avoid any contact of the needle tip with the implant and to monitor the correct injection of steroid solution between the capsule and the implant.

In our study, several limitations should be taken into account. First, we did not have a control group, our design being non-randomised. This seems particularly important, as future investigations should be focused on understanding whether the steroid is effective in the treatment of $\mathrm{CC}$ or the therapeutic effect is due to simple capsular distension by the injected solution. Second, we had a limited follow-up period. In our particular series, patients with breast expanders were then implanted with new textured prostheses and did not develop any new CC. Finally, the sample size was relatively small. This was particularly true for the LR (only five patients). Also, the study population was relatively heterogeneous, including both cancer and noncancer patients. However, to our knowledge this is the first reported experience of US-guided percutaneous treatment of $\mathrm{CC}$ in patients with augmented and reconstructed breast.

Summarising, US guidance allows for the safe injection of steroid into the small space around the implant and the US-guided injection of triamcinolone acetonide into the peri-implant pouch is effective in reducing $\mathrm{CC}$ after breast augmentation or reconstruction, even in patients who underwent adjuvant chemotherapy or radiation therapy.

\section{References}

1. Camirand A, Doucet J, Harris J (1999) Breast augmentation: compression - a very important factor in preventing capsular contracture. Plast Reconstr Surg 104:529-538

2. Siggelkow W, Faridi A, Spiritus K, Klinge U, Rath W, Klosterhalfen B (2003) Histological analysis of silicone breast implant capsules and correlation with capsular contracture. Biomaterials 24:1101-1109

3. Ersek RA (1992) Firestorm fibrosis: the fast fibrotic phenomenon. Ann Plast Surg 28:502

4. Embrey M, Adams EE, Cunningham B, Peters W, Young VL, Carlo GL (1999) A review of the literature on the etiology of capsular contracture and a pilot study to determine the outcome of capsular contracture interventions. Aesthet Plast Surg 23:197-206

5. Wong CH, Samuel M, Tan BK, Song C (2006) Capsular contracture in subglandular breast augmentation with textured versus smooth breast implants: a systematic review. Plast Reconstr Surg 118:1224-1236

6. Schlesinger SL, Ellenbogen R, Desvigne MN, Svehlak S, Heck R (2002) Zafirlukast (Accolate): a new treatment for capsular contracture. Aesthet Surg J 22:329-336

7. Shah Z, Lehman JA Jr, Tan J (1981) Does infection play a role in breast capsular contracture? Plast Reconstr Surg 68:34-42

8. Baker JL (1978) Augmentation mammoplasty. In: Owsley JQ, Peterson R (eds) Symposium on aesthetic surgery of the breast. Mosby, St Louis, pp 125-152

9. Behranwala KA, Dua RS, Ross GM, Ward A, A'hern R, Gui GP (2006) The influence of radiotherapy on capsule formation and aesthetic outcome after immediate breast reconstruction using biodimensional anatomical expander implants. J Plast Reconstr Aesthet Surg 59:1043-1051

10. Percec I, Bucky LP (2008) Successful prosthetic breast reconstruction after radiation therapy. Ann Plast Surg 60:527-531

11. Sugimoto T (1982) Open capsulotomy for capsular contracture: a new procedure for the prevention of recurrence. Aesthet Plast Surg 6:225-230

12. Little G, Baker JL Jr (1980) Results of closed compression capsulotomy for treatment of contracted breast implant capsules. Plast Reconstr Surg 65:30-33

13. Gruber RP, Jones HW (1981) Review of closed capsulotomy complications. Ann Plast Surg 6:271-276

14. Caffee HH, Rotatori DS (1993) Intracapsular injection of triamcinolone for prevention of contracture. Plast Reconstr Surg 92:1073-1077

15. Caffee HH (1994) Intracapsular injection of triamcinolone for intractable capsule contracture. Plast Reconstr Surg 94:824-828

16. Caffee HH (2002) Capsule injection for the prevention of contracture. Plast Reconstr Surg 110:1325-1328

17. Langley GB, Sheppeard H (1985) The visual analogue scale: its use in pain measurement. Rheumatol Int 5:145-148 